Journal of Mathematics and Informatics

Vol. 19, 2020, 125-133

ISSN: 2349-0632 (P), 2349-0640 (online)

Published 5 November 2020

Journal of

www.researchmathsci.org

DOI: http://dx.doi.org/10.22457/jmi.v19a11184

\title{
Numerical Solution of Singularly Perturbed Two Point Boundary Value Problems by Using Non-Polynomial Exponential Spline Functions
}

\author{
Ahmed R. Khlefha \\ Department of Statistics, University of Sumer, Alrifaee, Iraq. \\ Email: arkdsh85@gmail.com
}

Received 22 September 2020; accepted 31 October 2020

Abstract. This paper presents the application of non-polynomial Exponential spline method for finding the numerical solution of singularly perturbed boundary value problems. Two numerical examples are considered to demonstrate the usefulness of the method and to show that the method converges with sufficient accuracy to the exact solutions.

Keywords: Non-polynomial Exponential spline method, singularly perturbation, truncation error, exact solution.

\section{AMS Mathematics Subject Classification (2010): 41A15}

\section{Introduction}

Singular perturbation problems containing a small perturbation parameter, arise very frequently in many branches of applied mathematics such as,fluid dynamics, quantum mechanics, chemical reactor theory, elasticity, aerodynamics, and the other domain of the great world of fluid motion [1-3]

A well known fact is that the solution of such problems has a multiscale character ,i.e. there are thin transition layers where the solution varies very rapidly, while away from the layer the solution behaves regularly and varies slowly. Numerically, the presence of the perturbation parameter leads to difficulties when classical numerical techniques are used to solve such problems, this is due to the presence of the boundary layers in these problems.

We consider a second order singularly perturbed boundary problem [4-5]:

with the boundary

$$
\varepsilon y^{\prime \prime}+w(x) y^{\prime}+u(x) y=r(x), x \in[a, b]
$$

conditions

$$
\text { and } y(b)=\gamma_{2} y(a)=\gamma_{1}
$$

where $\varepsilon$ is a small positive parameter $0<\varepsilon<1, \gamma_{1}$ and $\gamma_{2}$ are given constants, $w(x)$, $u(x)$ and $r(x)$ are assumed to be sufficiently continuously differentiable functions. The non-polynomial Exponential spline method [6-16] developed in this paper has lower computational cost and its only requires solving $n+1$ linear or non-linear equations. 
Numerical Solution of Singularly Perturbed Two Point Boundary Value Problems by Using Non-Polynomial Exponential Spline Functions

\section{Derivation of the method}

We divide the interval $[a, b]$ into $n+1$ equal subintervals using the point

$$
x_{i}=a+i h, \quad i=0,1,2, \ldots, n, n+1 \text {, }
$$

With $a=x_{0}, b=x_{n+1}$ and $h=\frac{b-a}{n+1}$, where $n$ arbitrary positive integer.

Let $y(x)$ be the exact solution and $y_{i}$ be an approximation to $y\left(x_{i}\right)$ obtained by the non polynomial Exponential spline $E_{i}(x)$ passing through the points $\left(x_{i}, y_{i}\right)$ and $\left(x_{i+1}, y_{i+1}\right)$, we do not only require that $E_{i}(x)$ satisfies interpolatory conditions at $x_{i}$ and $x_{i+1}$ but also the continuity of first derivative at the common nodes $\left(x_{i}, y_{i}\right)$ are fulfilled. We write $E_{i}(x)$ in the form [7-8]:

$$
E_{i}(x)=a_{i} e^{k\left(x-x_{i}\right)}+b_{i} e^{-k\left(x-x_{i}\right)}+c_{i}\left(x-x_{i}\right)+d_{i}
$$

where $a_{i}, b_{i}, c_{i}$ and $d_{i}$ are constants and $k$ is free parameter to be determined later.

A non-polynomial Exponential spline function $E(x)$ of class $C^{2}[a, b]$ interpolates $y(x)$ at the grid points $x_{i}, i=0,1,2, \ldots, n+1$ depends on a parameter $k$, and reduces to ordinary spline $E(x)$ in $[a, b]$ as $k \rightarrow 0$.

To derive expression for the coefficient of equation (2) in term $y_{i}, y_{i+1}, F_{i}$ and $F_{i+1}$, we first define:

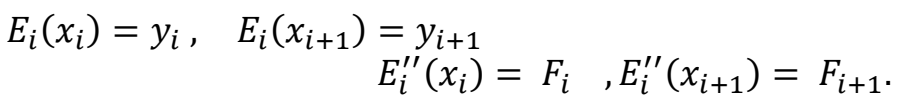

From algebraic manipulation, we get the following expression:

$$
\begin{gathered}
a_{i}=\frac{h^{2}\left(-F_{i} e^{-\theta}+F_{i+1}\right)}{\theta^{2}\left(e^{\theta}-e^{-\theta}\right)} \\
b_{i}=\frac{h^{2}\left(F_{i} e^{\theta}-F_{i+1}\right)}{\theta^{2}\left(e^{\theta}-e^{-\theta}\right)} \\
c_{i}=\frac{-h\left(F_{i+1}-k^{2} y_{i+1}+k^{2} y_{i}-F_{i}\right)}{\theta^{2}} \\
d_{i}=\frac{\left(\theta^{2} y_{i}-h^{2} F_{i}\right)}{\theta^{2}}
\end{gathered}
$$

where $\theta=k h$ and $i=0,1,2, \ldots, n$.

We applying the first derivative at $\left(x_{i}, y_{i}\right)$, that is $E_{i-1}^{\prime}\left(x_{i}\right)=E_{i}^{\prime}\left(x_{i}\right)$,gives the following consistency relation for $i=1, \ldots, n$ :

$y_{i-1}-2 y_{i}+y_{i+1}=\left(\frac{\left(\theta e^{-\theta}-2 \theta e^{\theta}+e^{2 \theta}+e^{-2 \theta}-2\right) h^{2}}{\theta^{2}\left(e^{2 \theta}+e^{-2 \theta}-2\right)}\right) F_{i-1}+$

$2\left(\frac{\left(\theta e^{2 \theta}-\theta e^{-2 \theta}-e^{2 \theta}-e^{-2 \theta}+2\right) h^{2}}{\theta^{2}\left(e^{2 \theta}+e^{-2 \theta}-2\right)}\right) F_{i}+$

$\left(\frac{\left(\theta e^{-\theta}-2 \theta e^{\theta}+e^{2 \theta}+e^{-2 \theta}-2\right) h^{2}}{\theta^{2}\left(e^{2 \theta}+e^{-2 \theta}-2\right)}\right) F_{i+1}$

which can further be written as

where

$$
\varepsilon\left(y_{i-1}-2 y_{i}+y_{i+1}\right)=h^{2} \varepsilon\left[\lambda\left(F_{i-1}+F_{i+1}\right)+2 \varepsilon \eta F_{i}\right]
$$

$\lambda=\frac{\left(\theta e^{-\theta}-2 \theta e^{\theta}+e^{2 \theta}+e^{-2 \theta}-2\right)}{\theta^{2}\left(e^{2 \theta}+e^{-2 \theta}-2\right)}$ 
Ahmed R. Khlefha

$\eta=\frac{\left(\theta e^{2 \theta}-\theta e^{-2 \theta}-e^{2 \theta}-e^{-2 \theta}+2\right)}{\theta^{2}\left(e^{2 \theta}+e^{-2 \theta}-2\right)}$

the local truncation errors,

$n=1,2, \ldots, \mathrm{n}-1$, in equation (6) can beobtained as follows: $t_{i}$

First we re-write the equation.(6) in the form ,

$$
\varepsilon\left(y_{i-1}-2 y_{i}+y_{i+1}\right)=h^{2} \varepsilon\left[\lambda y_{i-1}^{(2)}+2 \varepsilon \eta y_{i}^{(2)}+\lambda y_{i+1}^{(2)}\right]
$$

the terms $y_{i-1}^{(2)}, y_{i}^{(2)}$ and $y_{i+1}^{(2)}$ in equation (7) are expanded around the point ${ }^{x_{i}}$ using Taylor series and the expressions for $t_{i}, n=1,2, \ldots, \mathrm{n}-1$.can be obtained,

$$
t_{i}=h^{2}(1-2 \lambda-2 \eta) y_{i}^{(2)}+\frac{h^{2}}{12}(1-12 \lambda) y_{i}^{(4)}+\frac{h^{6}}{360}(1-30 \lambda) y_{i}^{(6)}+O\left(h^{8}\right)
$$

Now the (8) gives rise to the class of methods of different orders as follow:

Second order method:

For any choice of arbitrary $\lambda$ and $\eta$ with $=\frac{1}{6}, \eta=\frac{1}{3}$ and $\lambda+\eta=\frac{1}{2}$, then local truncation error is

$$
t_{i}=\frac{-h^{4}}{12} y_{i}^{(4)}+O\left(h^{6}\right), i=1,2, \ldots
$$

Fourth order method:

If $\lambda=\frac{1}{12}$ and $\eta=\frac{5}{12} \quad, T_{i}=O\left(h^{6}\right)$ then the resulting method is fourth order method.

Then the local truncation error is

$$
t_{i}=\frac{-h^{6}}{240} y_{i}^{(6)}+O\left(h^{8}\right), i=1,2, \ldots \mathrm{n}
$$

\section{Numerical scheme}

At the grid points $x$, Eq. (1) may be discretized by

$\varepsilon F_{i}+w(x) y^{\prime}+u(x) y=$

$$
r(x)
$$

Solving Eq (11) for $F_{i}$, we get

$$
\varepsilon F_{i}=-w(x) y^{\prime}-u(x) y+r(x)
$$

and approximate first derivative by using finite-difference.

The following approximation for the first-order derivative of $y$ in Eq. (12) can be used

$y^{\prime}{ }_{i-1}=\frac{-y_{i+1}+4 y_{i}-3 y_{i-1}}{2 h}$

$y^{\prime}{ }_{i}=\frac{y_{i+1}-y_{i-1}}{2 h}$

$y^{\prime}{ }_{i+1}=\frac{3 y_{i+1}-4 y_{i}+y_{i-1}}{2 h}$

So Eq. (12) becomes

$$
\begin{gathered}
\varepsilon F_{i-1}=-\frac{w\left(x_{i-1}\right)\left(-y_{i+1}+4 y_{i}-3 y_{i-1}\right)}{2 h}-u\left(x_{i-1}\right) y_{i-1}+r\left(x_{i-1}\right) \\
\varepsilon F_{i}=-\frac{w\left(x_{i}\right)\left(y_{i+1}-y_{i-1}\right)}{2 h}-u\left(x_{i}\right) y_{i}+r\left(x_{i}\right)
\end{gathered}
$$


Numerical Solution of Singularly Perturbed Two Point Boundary Value Problems by Using Non-Polynomial Exponential Spline Functions

$\varepsilon F_{i+1}=-\frac{w\left(x_{i+1}\right)\left(3 y_{i+1}-4 y_{i}+y_{i-1}\right)}{2 h}-u\left(x_{i+1}\right) y_{i+1}+r\left(x_{i+1}\right.$

Substituting Eq. (13)in Eq. (5), we get the following equation:

$\left[-\varepsilon+h \lambda\left(\frac{3 w\left(x_{i-1}\right)}{2}-\frac{w\left(x_{i+1}\right)}{2}\right)+h \beta w\left(x_{i}\right)-h^{2} \lambda u\left(x_{i-1}\right)\right] y_{i-1}+[2 \varepsilon+$

$\left.2 h \lambda\left(-w\left(x_{i-1}\right)+w\left(x_{i+1}\right)\right)-2 \eta h^{2} \lambda u\left(x_{i}\right)\right] y_{i}+\left[-\varepsilon+h \lambda\left(\frac{w\left(x_{i-1}\right)}{2}-\frac{3 w\left(x_{i+1}\right)}{2}\right)-\right.$

$\left.h \eta w\left(x_{i}\right)-h^{2} \lambda u\left(x_{i+1}\right)\right] y_{i+1}=-\lambda h^{2} r\left(x_{i-1}\right)-2 \eta h^{2} r\left(x_{i}\right)-$

$$
\lambda h^{2} r\left(x_{i+1}\right)
$$

\section{Stability analysis}

The tri-diagonal linear system (14) can be written in the following matrix form,

$$
A Y+h^{2} C R=G
$$

where $A=N+h B Q-h^{2} B u$ and $A$ is tri-diagonal and diagonally matrix of order $n-1$ Here $N=\left(L_{i j}\right)$ is a tri-diagonal matrix defined by are tri-diagonal matrices defined by

$L_{i j}=\left\{\begin{array}{cc}2 \varepsilon & i=j=1,2, \ldots, n-1 \\ -\varepsilon & |i-j|=1 \\ 0 & \text { othrwise }\end{array}\right.$

and $B Q=\psi_{i j}, B q=\Phi_{i j}$ are tri-diagonal matrices defined by

$\psi_{i j}=\left\{\begin{array}{lc}2 \lambda\left(-w\left(x_{0}\right)+w\left(x_{2}\right)\right), & i=j=1 \\ \lambda\left(\frac{3 w\left(x_{i-1}\right)}{2}-\frac{w\left(x_{i+1}\right)}{2}\right)-h \eta w\left(x_{i}\right), & i>j \\ 2 \lambda\left(\left(-w\left(x_{i-1}\right)+w\left(x_{i+1}\right)\right)\right. & i=j \\ \lambda\left(\frac{w\left(x_{i-1}\right)}{2}-\frac{3 w\left(x_{i+1}\right)}{2}\right)-h \eta w\left(x_{i}\right), & i<j \\ 2 \lambda\left(-w\left(x_{n-2}\right)+w\left(x_{n}\right)\right) & i=j=n-1\end{array}\right.$

And

$\Phi_{i j}= \begin{cases}2 \eta u\left(x_{i}\right) & i=j=1,2, \ldots, n-1 \\ \lambda u\left(x_{i-1}\right) & i>j \\ \lambda u\left(x_{i+1}\right) & i<j\end{cases}$

And

$$
Y=\left(y_{1}, y_{2}, \ldots, y_{n-2}, y_{n-1}\right)^{T} R=\left(r\left(x_{1}\right), r\left(x_{2}\right), \ldots, r\left(x_{n-2}\right), r\left(x_{n-1}\right)\right)^{T}
$$

The tri-diagonal matrix $C$ is defined by

where $G=\left(g_{1}, 0,0,0 \ldots, 0, g_{n-1}\right)^{T}$

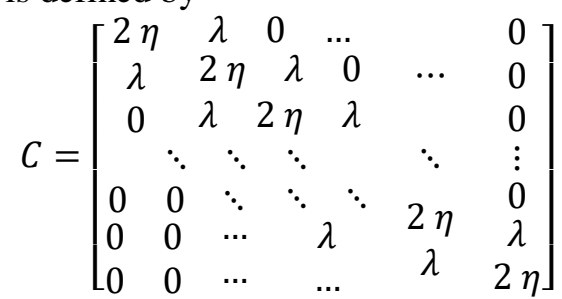

We assume that

$Y=\left(y_{1}, y_{2}, \ldots, y_{n-2}, y_{n-1}\right)^{T}$

Be the exact solution of the given boundary value problem (1) at nodal point $x_{i}$, for $i=$ $0,1,2, \ldots, n-1$ and then we have 
Ahmed R. Khlefha

$A \bar{Y}+h^{2} D R=T(h)+G$

If we subtract equation (12) from equation (13) we get the following

$A(\bar{Y}-Y)=A E=T(h)$

\section{Convergence analysis}

Our main purpose now is to derive a bound $\|\mathrm{E}\|$. We now turn back to the error equation in (13) and rewrite it in the form

$E=A^{-1} T=\left[N+h B Q-h^{2} B u\right]^{-1} T=\left[I+N^{-1}\left(h B Q-h^{2} B u\right)\right]^{-1} N^{-1} T$

$\|E\|_{\infty} \leq\left\|\left[I+N^{-1}\left(h B Q-h^{2} B u\right)\right]^{-1}\right\|_{\infty}\left\|N^{-1}\right\|_{\infty}\|T\|_{\infty}$

In order to derive the bound on $\|E\|_{\infty}$ the following two lemmas are needed.

Lemma 1. [17] If $G$ is a square matrix of order $n$ and $\|G\|<1$, then the $(I+G)^{-1}$ exists and $\left\|(I+G)^{-1}\right\| \leq(1-\|G\|)^{-1}$

Lemma 2: The matrix $\left(N+h B Q-h^{2} B u\right)$ is nonsingular $\|w\|_{\infty}<\frac{8 h \varepsilon}{(a-b)^{2}(8 \lambda+2 \eta)}$ and $\|u\|_{\infty}<\frac{8(1-\varepsilon)}{(a-b)^{2}} \quad$ where. $0<\varepsilon<1$

Proof:

Since, $A=\left(N+h B Q-h^{2} B u\right)=\left(I+N^{-1}\left(h B Q-h^{2} B u\right)\right) N$ and the matrix $N$ is nonsingular, so to prove $A$ nonsingular it is sufficient to show $\left(I+N^{-1}\left(h B Q-h^{2} B u\right)\right)$ nonsingular.

Since

$\left\|N^{-1}\left(h B Q-h^{2} B u\right)\right\|_{\infty} \leq\left\|N^{-1}\right\|_{\infty}\left(\left\|\left(h B Q-h^{2} B u\right)\right\|_{\infty}\right) \leq\left\|N^{-1}\right\|_{\infty}\left(\|h B Q\|_{\infty}+\right.$ $\left.\left\|h^{2} B u\right\|_{\infty}\right)$

Moreover,

$$
\begin{aligned}
& {[17]\left\|N^{-1}\right\|_{\infty}<\frac{(a-b)^{2}}{8 h^{2}}} \\
& \text { and }\left\|h^{2} B u\right\|_{\infty} \leq h^{2}\|u\|_{\infty} \text { and }\|h B Q\|_{\infty} \leq h(8 \lambda+2 \eta)\|w\|_{\infty}
\end{aligned}
$$

and $\|u\|_{\infty}=\max _{a \leq x \leq b}\left|u\left(x_{i}\right)\right|\|w\|_{\infty}=\max _{a \leq x \leq b}\left|w\left(x_{i}\right)\right|$

There for, substituting $\left\|N^{-1}\right\|_{\infty},\|h B Q\|_{\infty},\left\|h^{2} B u\right\|_{\infty}$, in equation (17) we get,

$$
\left\|N^{-1}\left(h B Q-h^{2} B u\right)\right\|_{\infty} \leq \frac{(b-a)^{2}}{8 h^{2}}(8 \lambda+2 \eta)\|w\|_{\infty}+\frac{(b-a)^{2}}{8}\|u\|_{\infty}
$$

Since,

$$
\left\{\begin{array}{c}
\|w\|_{\infty}<\frac{8 h \varepsilon}{(a-b)^{2}(8 \lambda+2 \eta)} \\
\|u\|_{\infty}<\frac{8(1-\varepsilon)}{(a-b)^{2}}
\end{array}\right.
$$

There for equations (20) and (21) leads to \|\|$u\left\|_{\infty}\left(h B Q-h^{2} B u\right)\right\|_{\infty} \leq 1$. From Lemma (1) it show that the matrix $A$ is nonsingular. Since $\left\|N^{-1}\left(h B Q-h^{2} B u\right)\right\|_{\infty} \leq 1$ so using Lemma (1) and equation (16) follow that

$$
\|E\|_{\infty} \leq \frac{\left\|N^{-1}\right\|_{\infty}\|T\|_{\infty}}{1-\left\|N^{-1}\right\|_{\infty}\left\|\left(h B Q-h^{2} B u\right)\right\|_{\infty}}
$$


Numerical Solution of Singularly Perturbed Two Point Boundary Value Problems by Using Non-Polynomial Exponential Spline Functions

\section{Numerical results}

We solve two singular perturbed problems using different values of $h$ and $\varepsilon$. The numerical solutions are computed and compared with the exact solutions at grade points. All calculations are implemented by Maple 13.

Example 1. Consider the following equation with variable coefficients

$$
\begin{aligned}
& \varepsilon y^{\prime \prime}+x y^{\prime}-y=-\left(1+\varepsilon \pi^{2}\right) \cos (\pi x)-(\pi x) \sin \pi x \\
& y(-1)=-1, y(1)=1
\end{aligned}
$$

The exact solution is given by:

$$
y(x)=\cos (\pi x)+x+\frac{x \operatorname{erf}\left(\frac{x}{\sqrt{2 \varepsilon}}\right)+\sqrt{\frac{2 \varepsilon}{\pi}} e^{-\left(\frac{x^{2}}{2 \varepsilon}\right)}}{x \operatorname{erf}\left(\frac{1}{\sqrt{2 \varepsilon}}\right)+\sqrt{\frac{2 \varepsilon}{\pi}} e^{-\left(\frac{1}{2 \varepsilon}\right)}}
$$

The numerical result of the example1 are presented in table 1 and figure 1 for different values subinterval $N$ and $\varepsilon=1 / 64$.

Example 2. Consider the following equation with variable coefficients $\varepsilon y^{\prime \prime}-\frac{1}{x} y^{\prime}-\left(1+x^{2}\right) y=x$

The exact solution is $y(x)=e^{\left(x^{2}\right)}$. The maximum absolute errors are tabulated in Table

\begin{tabular}{|c|c|c|c|c|c|}
\hline \multirow{2}{*}{$x$} & \multicolumn{4}{|c|}{ Numerical Sol. } & \multirow{2}{*}{ Exact Sol. } \\
\hline & $\mathrm{N}=16$ & $\mathrm{~N}=32$ & $\mathrm{~N}=64$ & $\mathrm{~N}=128$ & \\
\hline $\begin{array}{l}- \\
7 / 8\end{array}$ & -0.9190258015 & -0.9219199438 & -0.9237709378 & -0.9240415171 & -.9243033330 \\
\hline $\begin{array}{l} \\
6 / 8 \\
\end{array}$ & -0.6984560039 & -0.7035617854 & -0.7068294729 & -0.7073106180 & -.7077785740 \\
\hline $\begin{array}{l}- \\
5 / 8\end{array}$ & -0.3722043319 & -0.3783607642 & -0.3822747958 & -0.3828522232 & -.3834150957 \\
\hline$-\overline{4}$ & -0.009537748283 & -0.0038551921 & -0.0003641383 & -0.0001416495 & -0.000141523 \\
\hline $\begin{array}{l}- \\
3 / 8\end{array}$ & 0.3881155740 & 0.3845924696 & 0.3827696718 & 0.3825442896 & 0.3823396261 \\
\hline $\begin{array}{l} \\
2 / 8\end{array}$ & 0.7079360848 & 0.7077704103 & 0.7085372990 & 0.7087543527 & .7090026623 \\
\hline $\begin{array}{l}- \\
1 / 8\end{array}$ & 0.9384379783 & 0.9408096810 & 0.9435055183 & 0.9440534564 & 0.9446355666 \\
\hline 0 & 1.092840722 & 1.095498023 & 1.098469883 & 1.099075370 & 1.099715459 \\
\hline $1 / 8$ & 1.188481264 & 1.190838603 & 1.193508694 & 1.194053107 & 1.194635567 \\
\hline $2 / 8$ & 1.204935832 & 1.206883138 & 1.208472633 & 1.208735746 & 1.209002662 \\
\hline
\end{tabular}
2 for different values $\varepsilon$ and $h$.

Table 1: Numerical solution of Example 1 at different value of subintervals 
Ahmed R. Khlefha

\begin{tabular}{|l|l|l|l|l|l|}
\hline $3 / 8$ & 1.131158356 & 1.132521790 & 1.132618238 & 1.132502994 & 1.132339626 \\
\hline $4 / 8$ & 0.9998408258 & 1.000964472 & 1.000152356 & 0.9998016554 & .9993681064 \\
\hline $5 / 8$ & 0.8674062236 & 0.8685359797 & 0.8674978662 & 0.8670873438 & 0.8665849039 \\
\hline $6 / 8$ & 0.7927591290 & 0.7938125624 & 0.7929781403 & 0.7926384601 & .7922214264 \\
\hline $7 / 8$ & 0.8258581569 & 0.8265503836 & 0.8261169714 & 0.8259288994 & 0.8256966672 \\
\hline
\end{tabular}

Table 2: Numerical solution of Example 2 at different value of subintervals

\begin{tabular}{|l|l|l|l|l|l|}
\hline \multirow{2}{*}{$x$} & \multicolumn{4}{|c|}{ Numerical Sol. } & \multirow{2}{*}{ Exact Sol. } \\
\cline { 2 - 5 } & $\mathrm{N}=16$ & $\mathrm{~N}=32$ & $\mathrm{~N}=64$ & $\mathrm{~N}=128$ & \\
\hline 1 & 1.009050167 & 1.010040167 & 1.010050067 & 1.010050166 & 1.010050167 \\
\hline 2 & 1.039810774 & 1.040800774 & 1.040810674 & 1.040810773 & 1.040810774 \\
\hline 3 & 1.093174284 & 1.094164284 & 1.0941741839 & 1.094174283 & 1.094174284 \\
\hline 4 & 1.172510871 & 1.173500871 & 1.173510771 & 1.173510869 & 1.173510871 \\
\hline 5 & 1.283025417 & 1.284015417 & 1.284025317 & 1.284025416 & 1.284025417 \\
\hline 6 & 1.432329415 & 1.433319415 & 1.4333293149 & 1.433329414 & 1.433329415 \\
\hline 7 & 1.631316220 & 1.632306220 & 1.632316120 & 1.632316219 & 1.632316220 \\
\hline 8 & 1.895480879 & 1.896470879 & 1.896480779 & 1.896480878 & 1.896480879 \\
\hline 9 & 2.246907987 & 2.247897987 & 2.247907887 & 2.247907986 & 2.247907987 \\
\hline 10 & 2.7172818289 & 2.718271827 & 2.718281728 & 2.718281827 & 2.718281828 \\
\hline
\end{tabular}

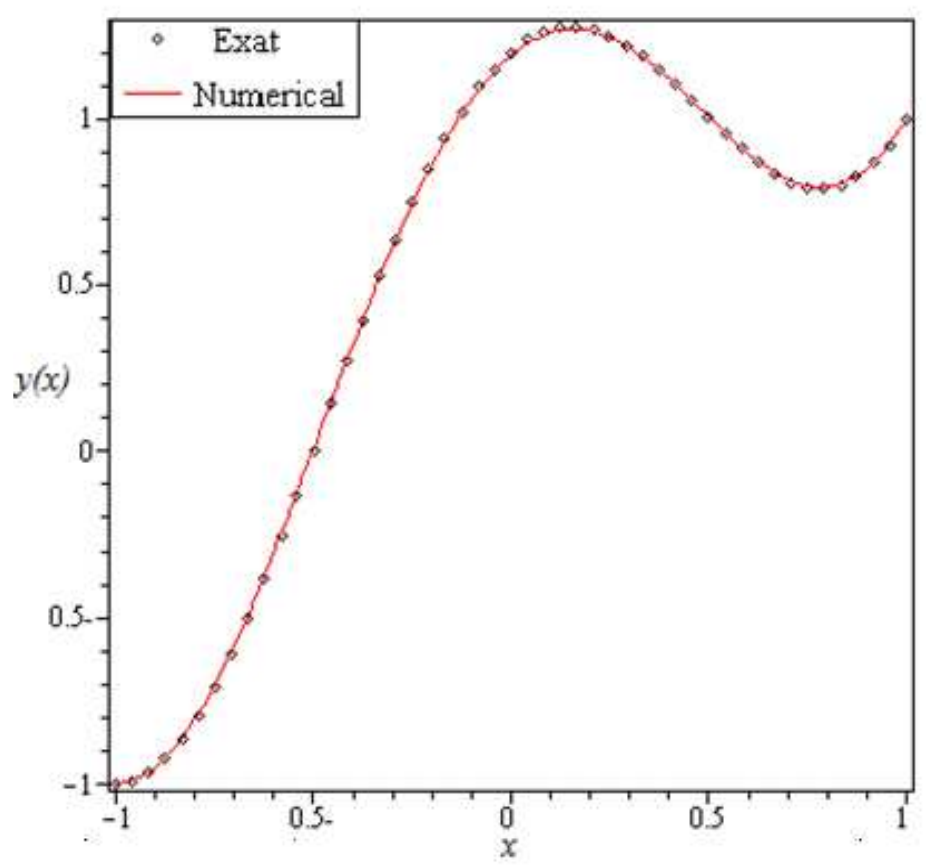

Figure 1: Comparison of exact and numerical solutions of example 1 for $N=\varepsilon=64$. 
Numerical Solution of Singularly Perturbed Two Point Boundary Value Problems by Using Non-Polynomial Exponential Spline Functions

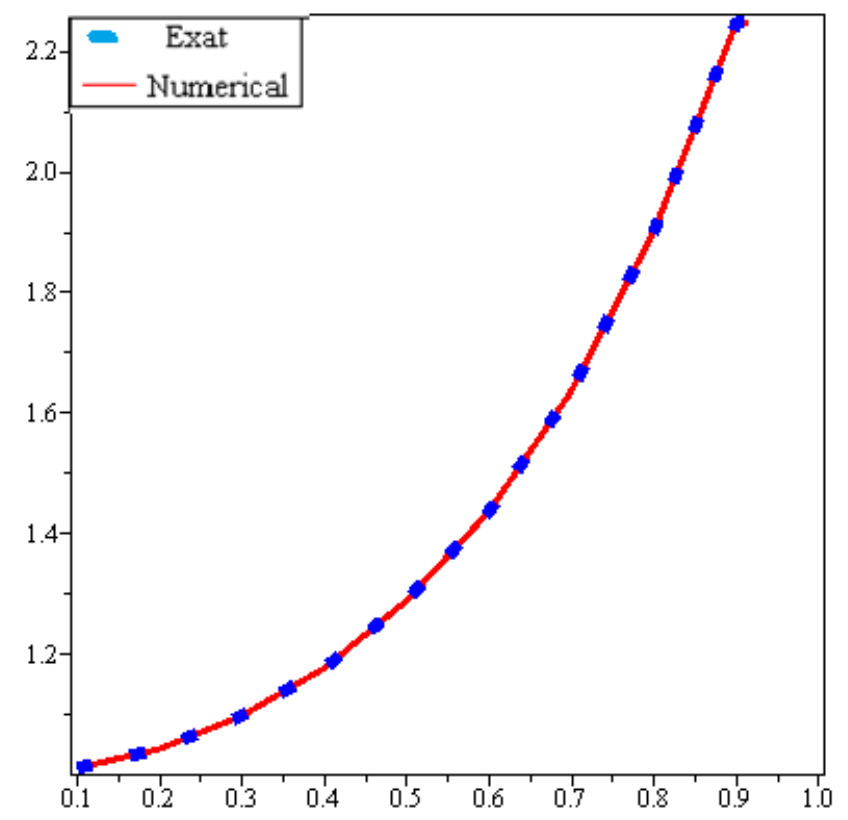

Figure 2: Comparison of exact and numerical solutions of example 2 for $N=\varepsilon=10$.

\section{7. conclusion}

in this paper, a numerical technique for singularly perturbed boundary value problems using non-polynomial exponential spline functions is derived. Simplicity of the adaptation of non-polynomial exponential spline and obtaining acceptable solutions can be noted as advantages of given numerical methods. The method is tested on two problems and the results obtained are very encouraging. The method is simple and easy to apply.

\section{REFFERENCES}

1. R.K.Bawa, Spline based computational technique for linear singularly perturbed boundary value problems, Appl. Math. Comput., 167 (2005) 225-236.

2. Z. Cen. Parameter-uniform finite difference scheme for a system of coupled singularly perturbed convection-diffusion equations, J. Sys. Sci. Complex., 18 (2005) 498-510.

3. A. Akrami, Numerical solution of singular perturbation two-point boundary value problems, Regional Conference on the Advance Math. and its App., 1 (2012) 65-73.

4. J.Yang, Alternative convergence analysis for a kind of singular perturbation boundary value problems, World Academy of Engineering and Technology, 52 (2011) 931-934.

5. M.Pal, Numerical Analysis for Scientists and Engineers: Theory and C Programs, Narosa Publishing House, New Delhi, (2007).

6. E.R. El-Zahar and S. M. El-Kabeir, A new method for solving singularly perturbed boundary value problems, App. Math. Inf. Sci., 7(3) (2013) 927-938.

7. K.Surla and M. Stojanovic, Solving singularly perturbed boundary value problems by spline in tension, J. of Computational and App. Math., 24 (2014) 355-363. 
Ahmed R. Khlefha

8. H.Caglar, C.Akkoyunlu, N.Caglar and D.Dundar, The numerical solution of singular two-point boundary value problems by using non-polynomial spline functions, In. conference on Applied computer and Applied computational Science, (2010) 23-25.

9. J.Surla, Solving singularly-perturbed boundary-value problems by splines in tension, J. Computational and Applied Mathematics, 24 (2018) 355-363.

10. J.Rashidinia and R. Mohammadi, Non-polynomial spline approximations for the solution of singularly-perturbed boundary value problems, TWMS J. Pure Appl. Math., 1(2) (2010) 236-251.

11. W. K.Zahra, F.A.Abd El-Salam, A.A.El-Sabbagh and Z. A. Zaki, Cubic nonpolynomial spline approach to the solution of a second order two-point boundary value problem, J. American Science, 6 (2010) 12.

12. E.A. Al-Said, M. A. Noor, A. H. Almualim, B.Kokkinis and J.Coletsos, Quartic spline method for solving second-order boundary value problems, In. J. Physical Sciences, 6(17) (2011) 4208-4212.

13. Li-Bin Lu, Y. Zhang and Huai-Huo Cao, Nonpolynomial spline difference schemes for solving second-order hyperbolic equations, In. J. Information Technology and Computer Science, 4 (2011) 43-49.

14. J. Rashidinia and M. Ghasemi, Cubic spline solution of singularly perturbed boundary value problems with significant first derivatives, Applied Mathematics and Computation, 190 (2007) 1762-1766.

15. J.Rashidinia and R. Mohammadi, Non-polynomial cubic splinemethods for thesolution of parabolic equations, In. J. of Computer Math., 85 (2008) 203-212.

16. S.Islam, Numerical solution of boundary- value problems using non-polynomial spline functions, Ph.D. Thesis, Ghulam Ishaq Khan Institute of Engineering Sciences and Technology (2005).

17. J.Rashidinia, R. Mohammadi and R. Jalilian, Cubic spline method for two point boundary value problems, International Journal of Engineering Science, 19 (2008) 3943. 